\title{
Theta Phase Classification of Interneurons in the Hippocampal Formation of Freely Moving Rats
}

\author{
András Czurkó, ${ }^{1,2,3}$ John Huxter, ${ }^{1,4}$ Yu Li, ${ }^{1,5}$ Balázs Hangya, ${ }^{6}$ and Robert U. Muller ${ }^{1,7}$ \\ ${ }^{1}$ Department of Anatomy, Medical School, University of Bristol, Bristol BS8 1TD, United Kingdom, ${ }^{2}$ Laboratory of Proteomics, Institute of Biology, Faculty \\ of Natural Sciences, Eötvös Loránd University, H-1117 Budapest, Hungary, ${ }^{3}$ Institute of Medical Chemistry, University of Szeged, 6720 Szeged, Hungary, \\ ${ }^{4}$ Pfizer Regenerative Medicine, Cambridge CB21 6GP, United Kingdom, ${ }^{5}$ School of Psychological Sciences, University of Manchester, Manchester M13 9PL, \\ United Kingdom, ${ }^{6}$ Laboratory of Cerebral Cortex Research, Department of Cellular and Network Neurobiology, Institute of Experimental Medicine, \\ Hungarian Academy of Sciences, H-1083 Budapest, Hungary, and 7Department of Physiology and Pharmacology, SUNY Downstate Medical Center, \\ Brooklyn, New York 11203
}

Earlier work on freely moving rats classified neurons in Ammon's horn as pyramidal cells (including place cells) or interneurons (previously called "theta cells") based on temporal discharge correlates and waveform configurations, but the anatomical and biochemical diversity of interneurons suggests they may have other distinguishing characteristics. To explore this possibility, we made extracellular recordings as rats foraged for food in an open space, used accepted criteria to identify interneurons, and found two additional categorization methods. First, interneurons were separated into theta-modulated and theta-independent groups using spike autocorrelograms. Second, theta-modulated interneurons were further separated into four groups by the phase of the $\sim 8 \mathrm{~Hz}$ theta rhythm at which firing was most rapid. These phase groups resemble the four phase peak groups of five anatomically identified interneuron types (two with the same preferred phase) recorded during the slow $(\sim 4 \mathrm{~Hz})$ theta rhythm in urethane-anesthetized rats. We suggest that the similar number of peak phase groups in walking rats and urethane-anesthetized rats and the partial agreement between peak phase values reflect a similar organization of theta rhythm in both states, so that the discharge properties of anatomically identified interneurons can be described in freely moving rats. Interestingly, the average spatial firing precision of the interneuron classes does not differ significantly, suggesting that the strong location-specific firing of place cells may be due to segregated high- and low-precision interneuron ensembles rather than to one or more dedicated high-precision classes.

\section{Introduction}

The discharge of principal cells in the hippocampal formation of freely moving rats (O'Keefe, 1979; Taube et al., 1990; Jung and McNaughton, 1993; Sharp, 1996; Hafting et al., 2005) provides a gateway for understanding the neural basis of navigation. The confinement of activity to cell-specific firing fields or angular ranges implies that neuronal activity reflects the operation of a map-like representation of the environment used to solve complex spatial problems (O'Keefe and Nadel, 1978).

It is clear, however, that principal cell activity cannot be separated from the mainly inhibitory actions of interneurons. Anatomically, hippocampal interneurons are a diverse group; their cell bodies are distributed throughout the layers of Ammon's horn, with widespread, varied dendritic trees and axonal ramifi-

\footnotetext{
Received Sept. 26, 2010; revised Dec. 17, 2010; accepted Dec. 23, 2010.

This work was supported by the Medical Research Council (United Kingdom) and National Institutes of Health Grant NS20686. We thank Dr. Jozsef Csicsvari for data analysis programs, the late Patrick D. Martin for acquisition programs, Chris Goodall for histological work, Matt Holzer and Julie Milkins for electronics and engineering support, and Bruno Rivard for developing and manufacturing the microdrives. We are also indebted to Dr. Szabolcs Kali for valuable discussions in regard to statistical analysis.

Correspondence should be addressed to Robert U. Muller, Department of Physiology and Pharmacology, SUNYDownstate Medical Center, 450 Clarkson Avenue, Brooklyn, NY 11203. E-mail: bob.muller@downstate.edu.

DOI:10.1523/JNEUROSCI.5037-10.2011

Copyright $\odot 2011$ the authors $\quad 0270-6474 / 11 / 312938-10 \$ 15.00 / 0$
}

cations (Amaral and Wittner, 1995; Freund and Buzsáki, 1996; Klausberger and Somogyi, 2008). Interneurons can be classified on molecular as well as anatomical grounds (McBain and Fisahn, 2001; Klausberger et al., 2003, 2004, 2005; Mott and Dingledine, 2003; Ferraguti et al., 2005; Somogyi and Klausberger, 2005; Baude et al., 2007; Jinno et al., 2007; Tukker et al., 2007; Fuentealba et al., 2008).

Hippocampal interneurons form complex networks that interact with subcortical and isocortical regions and with principal cells. Among network functions are control of overall hippocampal excitability, synchronization and generation of theta and gamma rhythms, and shaping the location specificity of primary cells. Given the diverse interneuron structure and connectivity, a full understanding of the hippocampal navigational system is possible only if the functional properties of each interneuron subtype are known.

An important step was made by Klausberger and colleagues (Klausberger et al., 2003, 2004; Ferraguti et al., 2005), who showed that anatomically identified interneurons discharge on four different phases of the slow $(3-4 \mathrm{~Hz})$ theta cycle characteristic of urethane anesthesia. This was done by recording interneuron firing in parallel with local field potentials and then doing juxtacellular labeling for subsequent visualization of the cells' structure. Unfortunately, it is currently implausible to apply this method to freely moving rats. 
To attempt to determine the anatomical identity of interneurons recorded in freely moving rats, we therefore recorded their activity and simultaneous local field potentials and calculated for each cell the theta phase at which it discharged most rapidly. Just as in urethane-anesthetized rats, we found that interneurons in walking rats fall into four different groups according to the peak theta phase of discharge. Moreover, the mean phase of two groups is very close to the highest excitability phase of groups seen during urethane theta. On this basis, we suggest a tentative anatomical classification of interneurons in freely moving rats. We also consider the functional significance of the spatial firing properties of the interneuron types. Separately, we report the existence of an interneuron class whose activity is remarkably constant over the entire theta cycle while occasionally discharging very high-frequency $(300-500 \mathrm{~Hz})$ bursts.

\section{Materials and Methods}

\section{Subjects}

The subjects were 17 male Long-Evans rats weighing 300-350 g before surgery. They were kept in individual cages on a 12:12 h light/dark cycle. All animal procedures were performed in accordance with the UK Animals (Scientific Procedures) Act 1986 and complied with guidelines for animal experimentation published in the National Institutes of Health publication Principles of Laboratory Animal Care. All efforts were made to minimize animal suffering and the number of animals used.

\section{Behavioral methods}

Recording sessions were $16 \mathrm{~min}$ in duration. Hungry rats (deprived to $85 \%$ ad libitum weight) chased $25 \mathrm{mg}$ food pellets randomly scattered at $\sim 3 /$ min into a $76-\mathrm{cm}$-diameter gray cylinder with a $45^{\circ}$ white cue card pasted on its wall at 3:00 as viewed from overhead. Recordings during pellet chasing are useful for studying the theta firing phase of interneurons, because trained rats spend most of their time locomoting in search of the food pellets, so the hippocampus is usually generating theta (Vanderwolf, 1969). The cylinder was surrounded by a 2-m-diameter dark circular curtain for visual isolation. The cylinder and curtain were centered in a $3.0 \mathrm{~m}$ by $3.0 \mathrm{~m}$ sound-attenuated room. Lighting was provided by eight $40 \mathrm{~W}$ bulbs evenly spaced at the perimeter of a 1-mdiameter disc suspended $2 \mathrm{~m}$ above the cylinder floor. During pellet chasing sessions, rats were tracked at $25 \mathrm{~Hz}$ with an overhead TV camera in a grid of $2.7 \mathrm{~cm} \times 2.7 \mathrm{~cm}$ pixels. To visualize spatial firing patterns, maps were constructed in which color encodes firing rate. In such maps, yellow indicates that a cell's firing rate was exactly zero. Increasing rates are coded in the following order: orange, red, green, blue, purple.

Surgery. Surgical procedures, electrode preparation, and implantation methods have been previously described (Muller et al., 1987; Fenton et al., 2000; Rivard et al., 2004; Huxter et al., 2007). In short, rats were implanted with four movable tetrodes for recording single-cell activity and local field potentials (EEG). Tetrodes were constructed from four 25 $\mu \mathrm{m} \mathrm{H}-\mathrm{ML}$ insulated Ni-Cr wires (Stablohm 675, California Fine Wire) twisted together. The tetrodes were held in a locally made implant that allowed each tetrode to be advanced separately. To mount the implant, the rat was anesthetized with isoflurane and put in a Kopf stereotaxic instrument. A midline cut was made in the scalp to expose the skull. A 2.0 - $\mathrm{mm}$-diameter hole was drilled in the skull centered at $-4.0 \mathrm{~mm}$ anteroposterior and $3.0 \mathrm{~mm}$ lateral. A $1.2-\mathrm{mm}$-diameter screw was inserted into the skull above the cerebellum on each side; these served as ground and electrical reference as well as to anchor the implant. An additional anchoring screw was placed in the skull near the front of the incision. The dura exposed by the hole was cut and the tetrode tips lowered to $1.8 \mathrm{~mm}$ below the brain surface (Paxinos and Watson, 1998). The implant was cemented to the rat skull with gentamicin-filled bone cement (Palacos R-40, Schering-Plough Europe).

Recordings. The electrodes were slowly advanced ( $60 \mu \mathrm{m}$ per day) until they reached CA1 stratum oriens as judged by the appearance of clear rhythmic activity in the $5-12 \mathrm{~Hz}$ theta band during walking and largeamplitude sharp waves during immobility and by detection of relatively large $(>100 \mu \mathrm{V})$ action potential waveforms. Subsequently, recordings were made during pellet chasing. Spikes were recorded from the four tetrodes and EEG activity was separately recorded from the same 16 channels ( 4 wires per tetrode) with different filter settings. For spikes, all four channels of a tetrode were digitized at $30 \mathrm{kHz}$ over an interval of 1.07 ms (32 samples per channel) when the amplitude on any wire exceeded a $100 \mu \mathrm{V}$ threshold; filtering was between $300 \mathrm{~Hz}$ and $6000 \mathrm{~Hz}$. The 16 tetrode wires were also used to continuously record local EEG digitized at $5 \mathrm{kHz}$; filtering was between $1 \mathrm{~Hz}$ and $475 \mathrm{~Hz}$.

Histology. Rats were killed with a sodium pentobarbital overdose and perfused transcardially with saline followed by $4 \%$ paraformaldehyde (PFA) in phosphate buffer. Brains were removed, stored in 4\% PFA, and transferred to $30 \%$ sucrose solution for $48 \mathrm{~h}$ before sectioning. Using a microtome, $30 \mu \mathrm{m}$ coronal sections were taken and stained with cresyl violet. On the coronal sections, positions of the electrode depths were confirmed by viewing the coronal sections with a microscope fitted with a calibrated reticle. Specifically, we noted the final depth of each tetrode track relative to stratum pyramidale of CA1.

Spike sorting. Spikes captured during pellet chasing were sorted into clusters (presumptive single cells) on the basis of their amplitude and shape on the tetrode channels. Spike clustering was done by a semiautomatic method. The initial sorting was done with an automatic classification program (KlustaKwik, developed by Kenneth D. Harris, Rutgers University, Newark, NJ) (Harris et al., 2000), followed by manual reassignment of the clusters based on cluster proximity, amplitude ratios, and cross-correlograms. This manual step was performed with gclust (Jozsef Csicsvari, University of Oxford, Oxford, UK) and with Off-line sorter, a 3D spike analysis program (Plexon). During the manual correction, autocorrelations of candidate clusters were inspected for spikes within the absolute refractory period. If such spikes were found, additional efforts were made to separate the waveforms. In addition, a crosscorrelation was computed for all cluster pairs. Given the relatively high firing rate of interneurons (Kubie et al., 1990; Freund and Buzsáki, 1996), spikes within the absolute refractory period are expected if the crosscorrelation is based on independent activity of two interneurons. The absence of such short interval spikes suggests mutual refractoriness so that the two clusters were merged if the averaged waveforms were nearly equal according to amplitude and shape.

Identification of bursts. Bursts were detected using autocorrelation and by inspection of raw data. An interneuron was classified as having burst characteristics if the autocorrelation had a maximum near $3.5 \mathrm{~ms}$ in awake rats or two maxima near 2 and $4 \mathrm{~ms}$ during sleep.

\section{Measuring spatial firing organization}

Spatial coherence (Kubie et al., 1990) was used to estimate the local smoothness of the spatial firing of interneurons. Coherence is calculated in two steps. In the first, the product-moment correlation is computed between the firing rate in each pixel and the average firing rate in the 8 nearest-neighbor pixels. Then, to normalize the correlation distribution, coherence is taken as the $z$-transform of the correlation. Spatial specificity was also assessed by maximal and average positional information (Olypher et al., 2003) and by information content per spike (Skaggs et al., 1993). We used $100 \mathrm{~ms}$ time windows for positional information calculations.

Detection of theta activity. Hippocampal EEG was recorded as local field potentials on tetrode wires referenced to cerebellar skull screws. Half-sine waves in the theta frequency range $(5-12 \mathrm{~Hz})$ were selected according to the time interval between successive negative- and positivegoing zero crossings that bracketed negative-going deflections in the EEG. The correlation between the half-sine waves and the EEG was computed and the interval accepted as theta if the correlation was $>0.85$. Negative theta peaks were used because positive peaks recorded from strata oriens and pyramidale were less distinct. Intervals between successive negative-going zero-crossings served to define a theta cycle, which was then divided into twenty $18^{\circ}$ bins, each of equal duration. The phase of each spike was determined from the time of its peak relative to the current theta cycle. A phase histogram was constructed for all spikes in a given cluster. The peak firing phase for a cluster was determined from the phase histogram by finding the three consecutive bins whose average height was greatest and taking the phase as the mean across these bins. To 


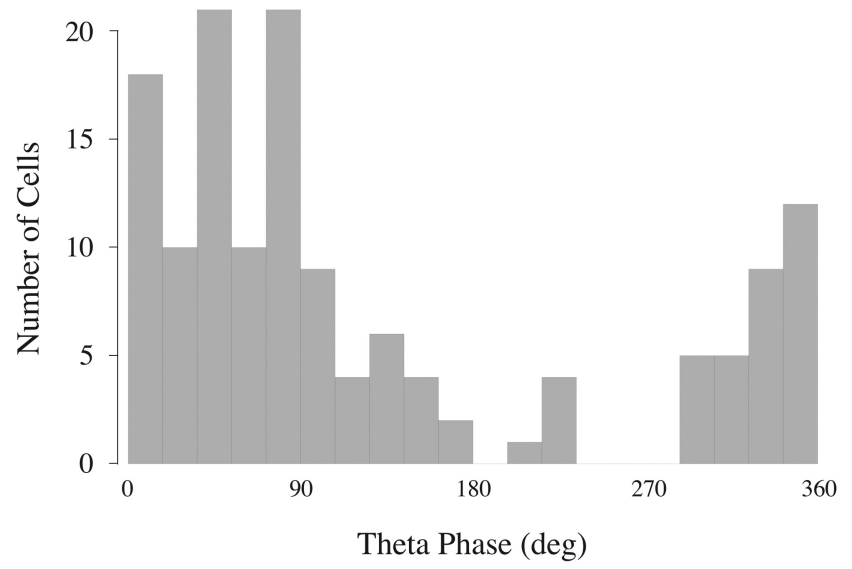

Figure 1. Histogram of the preferred theta firing phase of 141 identified pyramidal cells recorded along with the interneurons that are the main subject of this paper. The phase angles have been rotated $90^{\circ}$ clockwise from the values derived from our local EEG recordings so that they conform to the convention of Csicsvari et al. (1999). The resulting equivalence of the phase convention is visible by comparing this histogram to Figure $5 B$ of Csicsvari et al. (1999).

put our phase convention into register with the convention used in previous work of direct interest (Csicsvari et al., 1999; Klausberger et al., 2003,2004 ), we set phase-zero to the negative peak of the half-sine wave used to detect theta. As shown in Figure 1, the corrected distribution of preferred phases for pyramidal cells in our recordings is in good agreement with the distribution shown in Figure $5 B$ of Csicsvari et al. (1999). The same rotation has been applied to all phase angles reported in this paper. Population averages of phase values were calculated as circular means (Fisher, 1993).

Model selection for the distribution of preferred firing phases. In a first approach, we determined the number of modes in the phase histogram by fitting five models to the data assuming that the values are sampled from populations comprised of one, two, three, four, or five von Mises distributions. The von Mises distribution was chosen as the starting point for two reasons: (1) it is the circular analog of the Gaussian distribution and therefore a reasonable generating function for unimodal distributions on the circle; and (2) exact measures for the goodness of fit to sums of von Mises distributions are available. We found the best-fitting models with different numbers of components using an expectation maximization algorithm (Theodoridis and Koutroumbas, 2009, 2010) converted to von Mises instead of Gaussian distributions. We then tested the ability of these models to describe our sample using two different information theory measures applicable to model selection problems: Akaike information criterion (AIC) and Bayesian information criterion (BIC) (Akaike, 1974; Schwarz, 1978). Although the two measures are related, BIC more strongly penalizes the use of higher numbers of parameters.

To corroborate the information-theoretic method, we went on to implement a hypothesis testing approach described by Fisher (1993). In this algorithm, each combination of von Mises mixtures is tested against all others by simulating parametric resampling from the given model (bootstrap approach). Given that the best-fitting models with different number of von Mises components were tested, this procedure is equivalent to asking about the number of modes in the distribution. Best-fitting models were found by minimizing a least square criterion, based on residual differences between sample moments and corresponding fitted values [circular variant of the "method of moments" (Fisher, 1993)]. We tested the above five models performing 100 (50 for the model with five components) resampling steps.

\section{Results}

\section{Identification of interneurons}

The initial partitioning of CA1 neurons was into interneurons and "others," where the second category was composed almost exclusively of pyramidal cells, but also included a small number of slow-firing interneurons, according to waveform duration (see
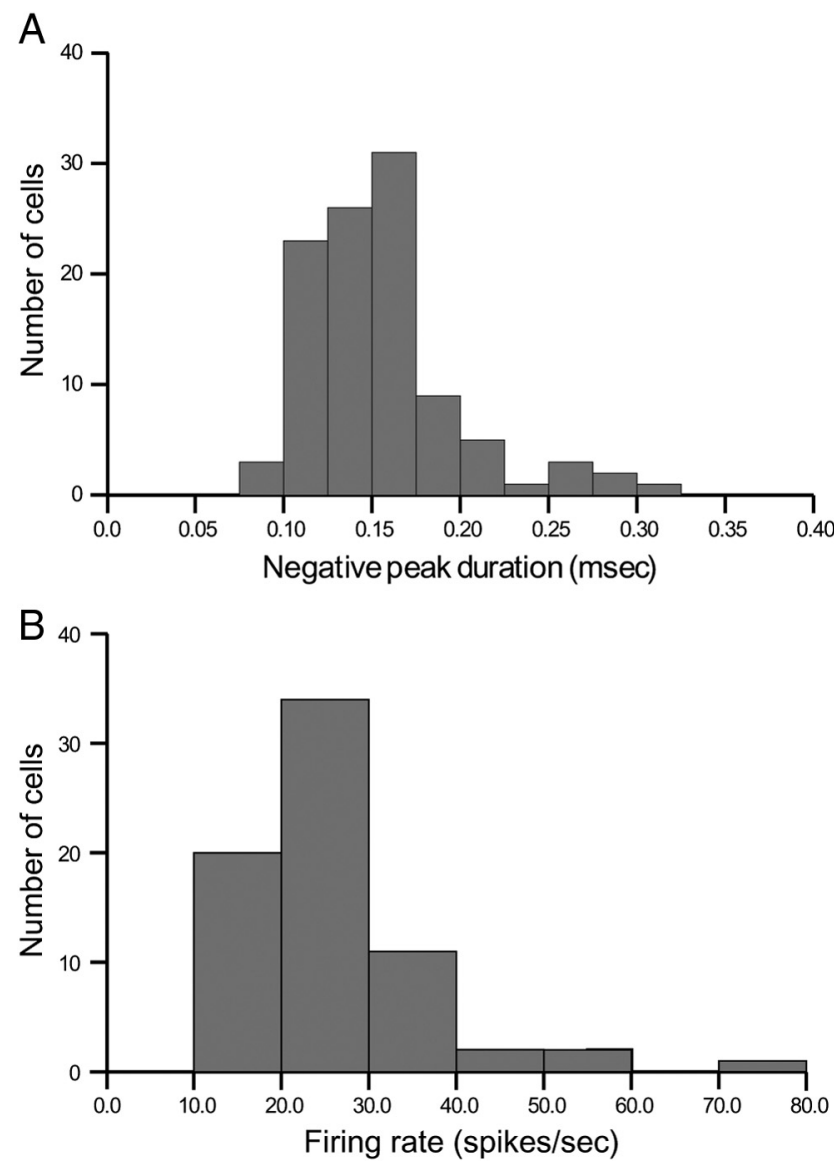

Figure 2. $\quad \boldsymbol{A}$, Histogram of the durations of the initial negative-going peak of interneuron spike waveforms. Classification according to negative-going peak duration was in excellent agreement with other criteria; there was a discrepancy only for the single cluster whose initial peak duration was $>300 \mu \mathrm{s}$. $\boldsymbol{B}$, Histogram of the overall firing rate for interneurons. The mean value was 20.8 spikes $/ \mathrm{s}$.

below). To be considered an interneuron, a cell had to meet the following criteria:

1. The duration of the initial negative phase of the extracellular action potential (approximation to the derivative of the rising part of the intracellular action potential) had to be brief (Fox and Ranck, 1981). We measured the duration at 1/e of the negative peak. The width had to be in the range 100-300 $\mu$ s. Only one of the candidates judged by other criteria to be an interneuron had an initial phase durations $>300 \mu \mathrm{s}$, as seen in the histogram of Figure $2 A$.

2. The overall firing rate of the cell during pellet chasing had to be $>5.0$ spikes/s. Previous work (e.g., Muller et al., 1987) indicates that the grand average rate of place cells in an open field is $\sim 1$ spike/s. By selecting the higher criterion rate, we excluded place cells and other cell types that discharge slowly during theta, including trilaminar and ivy cells (Ferraguti et al., 2005; Fuentealba et al., 2008). A histogram of overall firing rates for interneurons is shown in Figure $2 B$.

3 . In addition to average firing rate, a cell was considered an interneuron only if it never showed protracted $(>0.5 \mathrm{~s})$ silent intervals during pellet chasing. Place cells generate long silent intervals when the head is outside the firing field, whereas interneuron discharge may be slow but is never $<3-5$ spikes/s.

After applying these criteria, the sample size for subsequent analysis was 104 interneurons. 

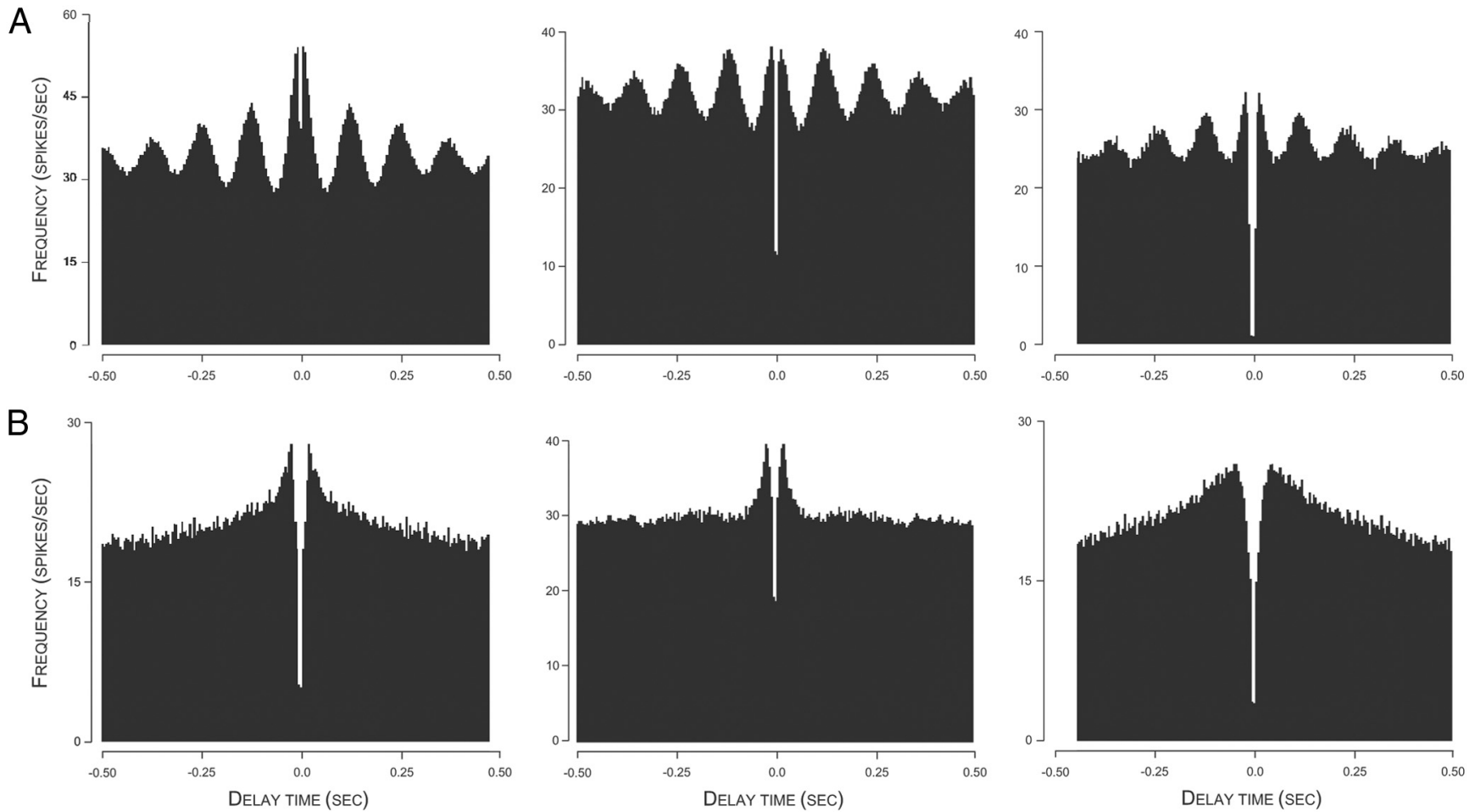

Figure 3. A, Autocorrelations for three theta-modulated interneurons. An approximate value for the $8 \mathrm{~Hz}$ frequency of local theta is visible from the four clear peaks for the $500 \mathrm{~ms}$ interval on each side of $t=0 . \boldsymbol{B}$, Autocorrelations for three theta-independent interneurons. Although the precise form of the autocorrelation function varies from cell to cell, there is no indication of theta-frequency modulation.

\section{Separation of cells into theta-modulated and}

theta-independent subtypes

By inspection, spike autocorrelograms for interneurons fell into one of two classes. One was characterized by prominent, equally spaced peaks of gradually decreasing amplitude. The mean frequency (1/interval) of these peaks was $8.27 \pm 0.00053 \mathrm{~Hz}$ (range $7.5-9.2 \mathrm{~Hz}$ ); these are referred to as "theta-modulated" cells. Three example autocorrelograms for theta-modulated interneurons are shown in Figure 3A.

The second class of interneurons is referred to as theta independent. For these cells, the autocorrelogram decreases monotonically as the delay increases from zero. Examples of autocorrelograms for theta-independent cells are shown in Figure $3 B$.

To formalize the distinction between the two cell types, we calculated an "oscillation score," which is $1-r$, where $r$ is the product-moment correlation between a straight line and successive values in the autocorrelogram. Thus, theta-modulated cells will have a high score, whereas theta-independent cells will have a low score. A histogram of the oscillation score (Fig. 4) shows a clear separation of the interneuron sample into two modes. By choosing an oscillation score of 0.45 , the sample consists of 74 theta-modulated and 30 theta-independent cells. In agreement with classification according to oscillation score, we find that all 74 theta-modulated cells have a (highly) significant Rayleigh vector for discharge relative to theta phase, whereas the Rayleigh vectors for the theta-independent cells are not significant (Huxter et al., 2008). Finally, we calculated for the discharge of each cell the peak power in the $5-12 \mathrm{~Hz}$ theta band cell normalized by the total power from $2-5 \mathrm{~Hz}$ plus $12-20 \mathrm{~Hz}$. The median value was 2.67 for 74 theta-modulated cells and 1.07 for 30 thetaindependent cells. According to a Mann-Whitney $U$ test, the probability of this outcome by chance is $\ll 0.0001$.

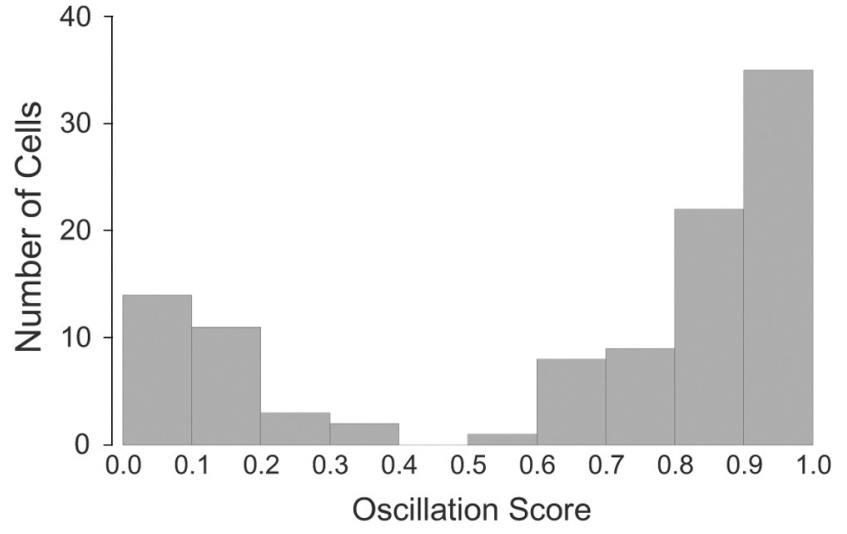

Figure 4. Histogram of oscillation score values (defined in text) for 104 interneurons. The distribution is strongly bimodal such that there are no values in the range $0.4-0.5$. Choosing a threshold of 0.45 thus separates the sample into two distinct groups such that high values are for theta-modulated cells and low values are for theta-independent cells.

\section{Differences between theta-independent and theta-modulated interneurons}

We next compared the two interneuron types and found additional differences. First, the anatomical location of cells in the two classes was distinct. The depth of a tetrode when a cell was recorded was estimated relative to the appearance of place cells as the tetrode moved. Place cells were never seen when a tetrode was in its original implanted position. As it advanced, place cells appeared, grew common, and then were no longer seen. The middle of this range was taken as the mid-depth of stratum pyramidale. Estimates of $d_{\mathrm{r}}$, the recording depth, made in this way were in good agreement with estimates using histological measurement of the final tetrode depth $d_{\mathrm{f}}$. Thus, $d_{\mathrm{r}}=d_{\mathrm{f}}-t \times p$, where $t$ is the 
number of screw turns after the recording and $p$ is the pitch of the screw. We used this depth estimate to divide interneurons into superficial (alveus/oriens), middle position (pyramidale), or deep (radiatum).

According to this classification, two-thirds of thetaindependent cells were in alveus/oriens, and except for one deep cell, the remainder were in stratum pyramidale. In contrast, theta-modulated cells were absent from alveus/oriens, 63/74 were found in stratum pyramidale, and 11/74 were found in stratum radiatum. The probability that the depth distributions are the same is $<0.0001$ according to a $\chi^{2}$ test. Thus, theta-independent cells occur mainly in superficial CA1, whereas theta-modulated cells occur in pyramidale and deeper. Although this is a relatively crude localization of presumed cell bodies, it nevertheless provides clues about the histological identities of theta-independent and theta-modulated cells.

We found that cells distinguished according to theta modulation also differ in average duration of the negative-going portion of the waveform. The median of this first deflection was $159 \mu \mathrm{s}$ for theta-modulated cells and $121 \mu$ s for theta-independent cells, a highly reliable difference according to a Mann-Whitney $U$ test $(p=0.000163)$. A histogram (not shown) for the duration of the first spike deflection shows a small second mode (centered on 276 $\mu \mathrm{s}$ ) whose six members are all theta modulated, perhaps indicating a subtype. When this mode is removed, the statistical difference of first deflection duration is preserved according to a second Mann-Whitney $U$ test $(p=0.00077)$. It is possible that some theta-independent cells with brief action potentials $(<100$ $\mu \mathrm{s}$ ) were fibers of passage, and indeed some of their shapes were triphasic with an initial positive-going deflection. Nevertheless, the presence of high-frequency discharge (see below) suggests that these may be the "bursty cells" reported by Csicsvari et al. (1999).

We also tested for differences in firing properties. The firing rate averaged over entire sessions was the same for both cell types; the mean rate for 74 theta modulated cells was 19.25 spikes/s versus 17.99 spikes/s for 30 theta-independent cells (MannWhitney $U$ test, $p=0.68$ ). The firing rate of both types increased when the hippocampal EEG was in the theta state compared to during the non-theta activity typically accompanied by sharp waves. The firing rate of modulated cells was $21.39 \pm 1.47$ spikes/s during theta and $19.04 \pm 1.40$ spikes/s during non-theta; the median ratio was 1.10 . Since the firing rate ratio in theta to non-theta was $>1.0$ for $65 / 74$ theta-modulated cells, a binomial test rejects the possibility that the rate was the same in both states ( $p \ll 0.0001)$. For theta-independent cells, the rate was 20.49 spikes/s during theta and 19.74 during non-theta with a median ratio of 1.046 . The firing rate ratio in theta compared to nontheta was $>1.0$ for $23 / 30$ cells, so that according to a binomial test, the probability that the rate is the same in both states is 0.0007. Thus, both classes of cells speed up when the EEG switches to the theta state, but according to a Mann-Whitney $U$ test, the normalized increase is larger for theta-modulated cells $(p=0.00075)$.

Given the strong tendency of theta to occur during locomotion and for non-theta to be present during quiet alertness or eating (Vanderwolf, 1969), firing rates should be higher during walking than otherwise. To test this prediction, the rat's speed for $0.4 \mathrm{~s}$ intervals was binned in the range $0.0-20.0 \mathrm{~cm} / \mathrm{s}$. The discharge rate was then calculated for each speed bin normalized for each cell by its discharge rate during quiet alertness $(0.0 \mathrm{~cm} / \mathrm{s})$. As expected from the variation of firing rate with EEG state, discharge for both theta-modulated and theta-independent cells in-

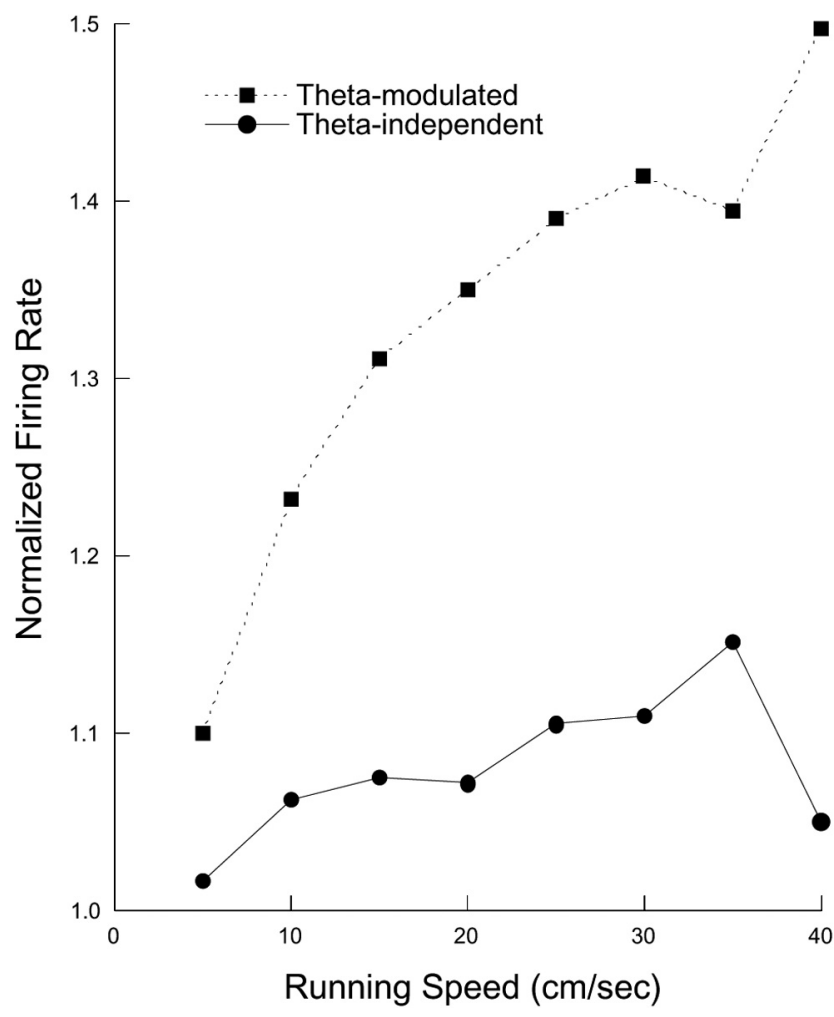

Figure 5. Firing rate as a function of running speed for theta-modulated and thetaindependent interneurons. The activity of both cell types increases with running speed, but the effect is greater for theta-modulated cells as confirmed by a significant group by running speed interaction in an ANOVA.

creased as the rat ran faster. Moreover, the increases were greater for theta-modulated cells (Fig. 5). A two-factor repeatedmeasure ANOVA with theta modulation type as one factor and running speed as the repeated measure confirmed these conclusions. The $F$-ratio was high for cell type $\left(F_{(1,102)}=36.2 ; p \ll\right.$ $0.0001)$, running speed $\left(F_{(8,816)}=46.3 ; p \ll 0.0001\right)$, and the type by speed interaction $\left(F_{(8,816)}=11.4 ; p \ll 0.0001\right)$.

Pairwise post hoc analyses conform closely to expectations from Figure 5. First, the normalized rate increase was significantly greater than the null-hypothesis value of 1.0 for both cell types at almost all running speeds; the exception was for thetaindependent cells at the highest running speed. Second, firing rate reached an asymptote for theta-independent cells at running speeds $>2.5 \mathrm{~cm} / \mathrm{s}$ and was little changed for the speed range $5.0-20.0 \mathrm{~cm} / \mathrm{s}$. In contrast, the firing rate for theta-modulated cells increased reliably with running speed up to $15.0 \mathrm{~cm} / \mathrm{s}$, above which there was little change.

Another difference is that the positional firing patterns of theta-modulated cells are more highly organized than those of theta-independent cells. The median spatial coherence was 0.162 (mean $=0.209 \pm 0.020)$ for theta-modulated cells and 0.0795 (mean $=0.100 \pm 0.031)$ for theta-independent cells. A MannWhitney $U$ test revealed that this difference was highly reliable ( $p=0.0053)$. According to Mann-Whitney tests, other possible differentiators between theta-modulated and theta-independent cells failed to distinguish the two types: maximal positional $-p=$ 0.79 ; average positional information $-p=0.27$; information content per spike $-p=0.77$. Nevertheless, the higher spatial coherence suggests that theta-modulated cells are more intimately associated with the spatial mapping activities thought to be among the most important functions of the rat hippocampus. 
A
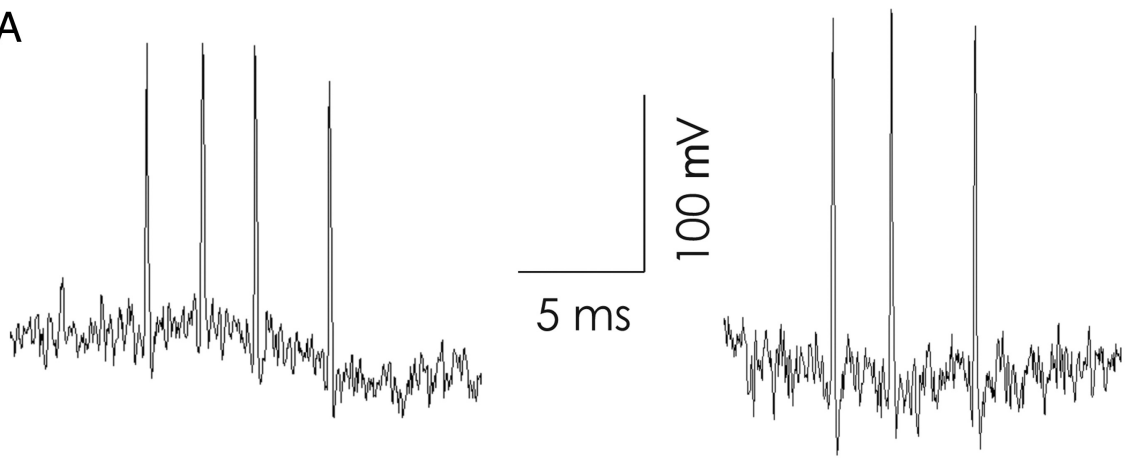

B

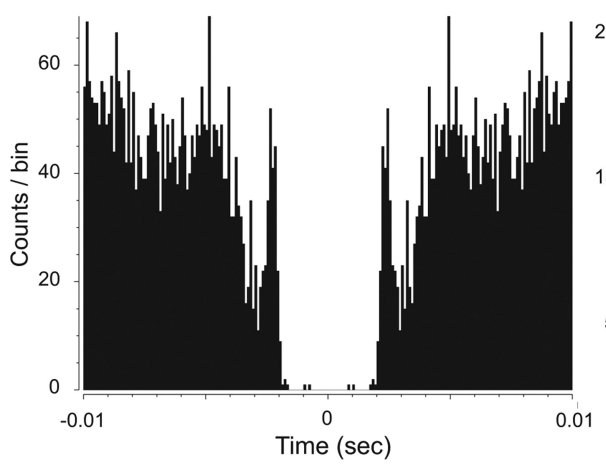

$\mathrm{C}$

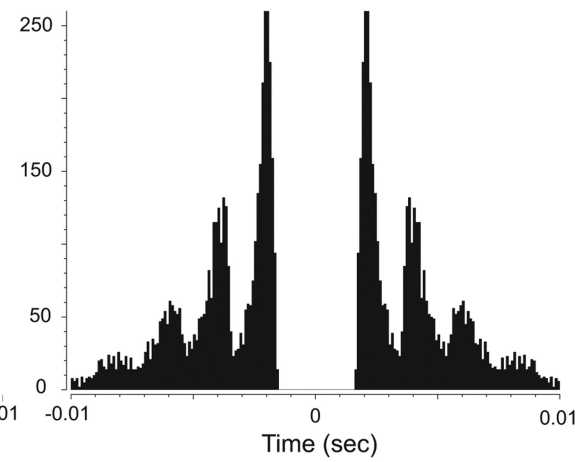

Figure 6. High-frequency burst activity by theta independent interneurons. $\boldsymbol{A}$, High-frequency burst generated by a thetaindependent interneuron during pellet chasing. Component spikes of the burst were acquired separately in 1.07 ms frames and then properly aligned from the time stamps to reproduce the intervals. $\boldsymbol{B}$, Autocorrelation for spikes acquired during pellet chasing. There is a clear mode at a delay of $\sim 3.5 \mathrm{~ms}(\sim 285 \mathrm{~Hz})$. C, Autocorrelation for spikes acquired during slow-wave sleep. The first mode is at $\sim 1.8 \mathrm{~ms}(\sim 514 \mathrm{~Hz})$. Additional modes at about 3.6 and $5.4 \mathrm{~ms}$ are also present. By inspection of the raw data, many of the 3.6 and 5.4 ms intervals were due to bursts of $>2$ spikes. The great difference in the scaling of the $y$ axes of $\boldsymbol{B}$ and $\boldsymbol{C}$ indicate that high-frequency activity was much more common during slow-wave sleep than during pellet chasing.

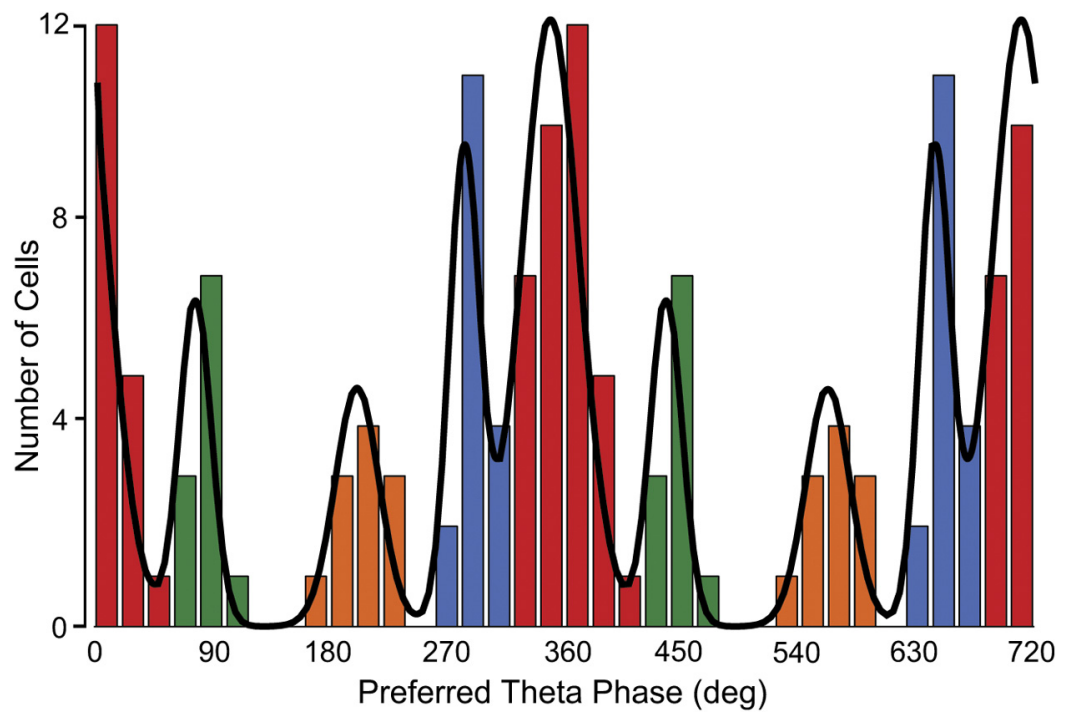

Figure 7. Histogram of preferred theta firing phases (two cycles are shown) for 74 theta-modulated interneurons. The color code emphasizes that there are four preferred phase modes. In this code, class 1 is red, class 2 is green, class 3 is orange, and class 4 is blue. The continuous line represents the sum of fits to four von Mises distributions whose means and dispersions are stated in the text. Note that the blue bin between $300^{\circ}$ and $320^{\circ}$ contains one cell from class 1 . A remarkable feature of the distribution is the isolation of class 3 cells from the others.

Theta-independent interneurons generate very highfrequency spike bursts

Most (25/30) of the theta-independent interneurons occasionally discharged high-frequency $(\sim 350 \mathrm{~Hz})$ bursts of two, three, or four spikes during pellet chasing; example bursts are shown in Figure $6 \mathrm{~A}$. Many of the theta-independent interneurons were also recorded during slow-wave sleep, during which they mainly discharged bursts of even higher $(\sim 500 \mathrm{~Hz})$ frequency. The peaks in the autocorrelograms for spikes recorded during walking (Fig. 6B) and slowwave sleep (Fig. 6C) demonstrate the strong tendency for rapid discharge.

\section{Subclassification of}

theta-modulated interneurons

To distinguish possible subtypes of thetamodulated interneurons, we followed one of the key methods of Klausberger and colleagues (summarized by Klausberger and Somogyi, 2008) and generated a thetaphase histogram for individual spikes fired by each of the 74 cells. We then determined the preferred firing phase for each cell and finally constructed the "preferred phase histogram" of Figure 7. To determine the number of modes in the preferred phase distribution, we fitted five models to the histogram assuming that the counts are sampled from a mixture of one, two, three, four, and five von Mises distributions (i.e., the circular analogs of Gaussians). We asked which model best describes our data using two different information theory measures, namely, AIC (Akaike, 1974) and BIC (Schwarz, 1978); for each measure, the lowest value is associated with the best model. According to both measures, the mixture of four von Mises distributions was the best fit (AIC values for models with one to five modes: 245.33, 251.25, 235.39, 221.74, 231.51; BIC values for models with one to five modes: 249.94, 262.77, 253.82, 247.09, 263.77). To corroborate the information theoretic analysis, we implemented a hypothesis testing method (Fisher, 1993). Models with one $(p=0.01)$ or two $(p \approx$ $0)$ modes were rejected. Models with three $(p=0.07)$ or five $(p=0.06)$ modes had probabilities above $\alpha=0.05$ and were not rejected. Nevertheless, the four-mode model had the highest probability of the tested cases $(p=0.15)$. Thus, this method also indicates that the mixture of four von Mises distributions provides the best description of the empirical preferred phase distribution with its four prominent modes.

Once it was clear that the distribution of preferred phases is best fit by four distinct types (as suggested by the appearance of Fig. 7), each cell was assigned to a class determined by maximal likelihood; the class identities are made explicit by the color code in Figure 7. The same color code is used in Figure 8, where an example is given of the spike theta- 


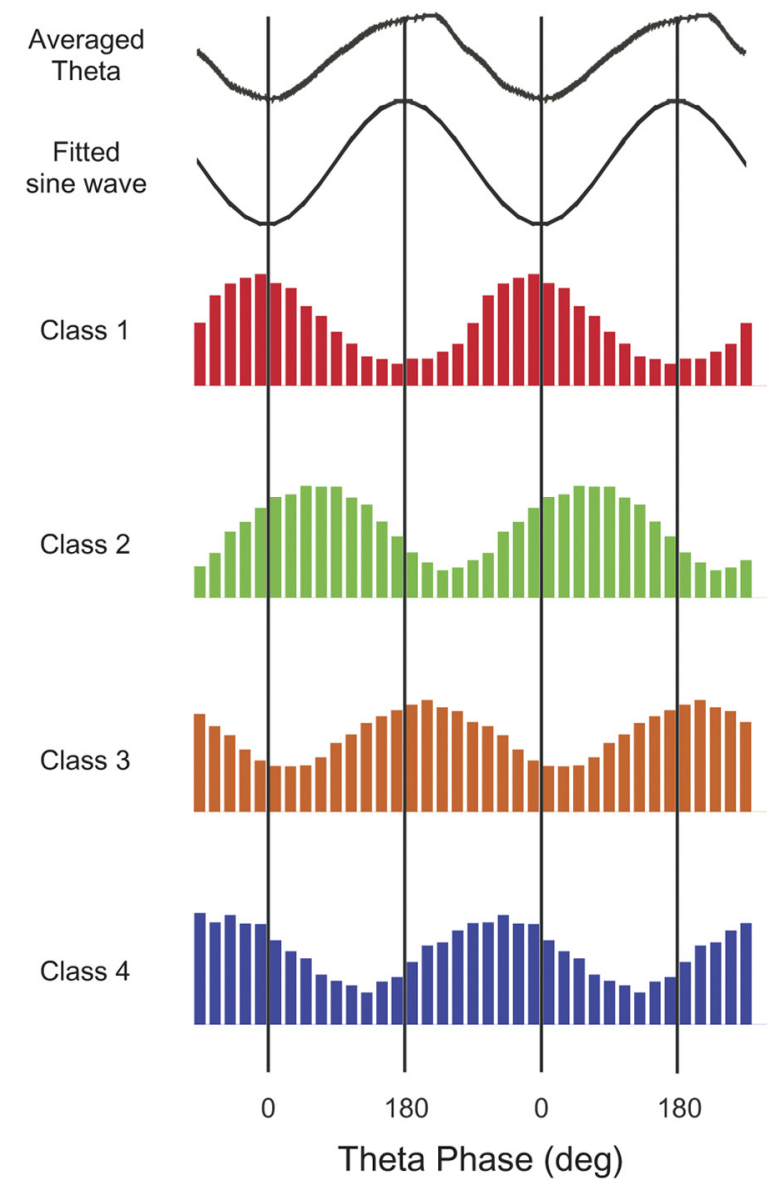

Figure 8. Phase histogram examples for one interneuron selected from each of the four preferred phase classes; the color code is the same as in Figure 7. The histograms are binned at $20^{\circ}$. The top panel shows the filtered, averaged theta activity recorded at the same time as the class 1 cell. The fitted sine wave shows the sine wave that would have been chosen to represent the negative half of the theta cycle. The preferred phase (middle of the preferred bin) and number of spikes for the four examples are as follows: class $1-350^{\circ}, 590$ spikes; class $2-50^{\circ}$, 1082 spikes; class $3-210^{\circ}, 2110$ spikes; class $4-310^{\circ}, 1532$ spikes.

phase histogram for each of the four classes aligned under an example of the raw hippocampal EEG and the sine wave used to measure the phase of each spike.

Class 1: This group of 36 interneurons (coded red) fired near the negative peak of theta oscillations with preferred phases between $310^{\circ}$ and $60^{\circ}$. The mean phase was $350.6^{\circ} \pm 19.8^{\circ} \mathrm{SD}$.

Class 2: The 11 cells in this group (coded green) preferentially fired on the rising portion of the theta rhythm between the negative and positive peaks; the phase range was between $60^{\circ}$ and $120^{\circ}$. The mean phase was $77.8^{\circ} \pm 12.5^{\circ} \mathrm{SD}$.

Class 3: The phase preferences for the 11 cells of this type (coded orange) are just after the positive theta peak and are clearly separated from the other classes; the theta phase range was between $160^{\circ}$ and $240^{\circ}$. The mean phase was $201.5^{\circ} \pm 18^{\circ} \mathrm{SD}$.

Class 4: The preferred theta phases for the 16 class 4 cells (coded blue) fell between $260^{\circ}$ and $310^{\circ}$, before the negative peak of the theta cycle. The mean phase was $284.1^{\circ} \pm 8^{\circ}$ SD.

To seek further distinctions among phase classes, we inspected rate maps to see whether any particular spatial firing pattern type was more common in a given theta-modulated class. As suggested by the examples in Figure 9, we were able to find cells in each class whose spatial firing patterns were organized to quite different extents. More formally, we did separate ANOVAs of coherence, average positional information, maximal positional information, information content per spike, and overall firing rate or firing rate when the EEG showed theta versus non-theta. We saw no reliable main cell class effect for any of these measures ( $p=0.79,0.33,0.23,0.25$, and 0.24 , respectively). We also failed to find a contingency between theta phase class and depth of the recording $\left(\chi_{(3)}^{2}=4.78, p=0.19\right)$.

\section{Firing rate varies with running speed for all phase classes}

We showed above that firing rate varies as a function of running speed for theta-modulated cells regardless of phase class. A similar relationship is seen for each of the phase classes (Fig. 10). A repeated-measure ANOVA done for running speed and group reveals a clear main speed effect but no group effect or speed by group interaction. Thus, the average discharge of each class depends in the same way on the intensity of locomotor activity. Since theta frequency varies slowly with running speed (Buzsáki et al., 1982; Czurkó et al., 1999; Huxter et al., 2007), the inference is that interneurons on the average fire more spikes per theta cycle.

\section{Phase classes and the anatomical identity of interneurons}

The observation that interneurons fall into four classes according to their preferred firing phase during walking theta is similar to the classification into anatomical types, accomplished by recording cells during low-frequency, urethane-induced theta and then using dye ejection from juxtacellular electrodes to stain each cell (Klausberger et al., 2003, 2004, 2005). The relationship between the two classification schemes is shown in Figure 11 by two sets of radial lines. The solid lines show the preferred angle for phase classes in walking rats, using the same, arbitrary color code as in Figures 7, 8, and 10. The dashed radial lines show the preferred angle for phase classes in urethane-anesthetized rats; each dashed line is labeled with the anatomical class. The proposed correspondence is based on the assumption that the identical phase sequence is followed by the local field potentials in walking and urethane-induced theta, with no attempt to rotate one phase sequence against the other. The fit of class 2 interneurons to oriens/ lacunosum-moleculare + bistratified cells is quite good, as is the agreement between class 3 interneurons and axo-axonic cells. The correspondences between class 2 and CCK cells and class 4 and PV basket cells are weaker, but it is interesting that rotating the anatomical classification against our classification in either direction tends to reduce the overall agreement between preferred phases in the two kinds of theta. Figure 11 therefore summarizes our proposed scheme for anatomically identifying interneurons in freely moving rats according to the scheme stated in Klausberger and Somogyi (2008).

\section{Discussion}

Hippocampal interneurons recorded from alert, freely moving rats fall into two broad classes, those whose discharge is modulated by the prominent theta rhythm and those whose firing is quite constant over the entire theta cycle. We treat thetamodulated cells first and then consider theta-independent cells.

\section{Theta-modulated interneurons}

Anatomically, hippocampal interneurons can be divided into many classes (Freund and Buzsáki, 1996) and into additional classes according to whether cells express certain molecules (McBain and Fisahn, 2001; Baude et al., 2007; Fuentealba et al., 2008). An initial functional classification was made by determining the pattern of interneuron discharge relative to sharp-wave activity during sleep (Csicsvari et al., 1999). We tried a similar method by recording during slow-wave sleep and pellet chasing but 

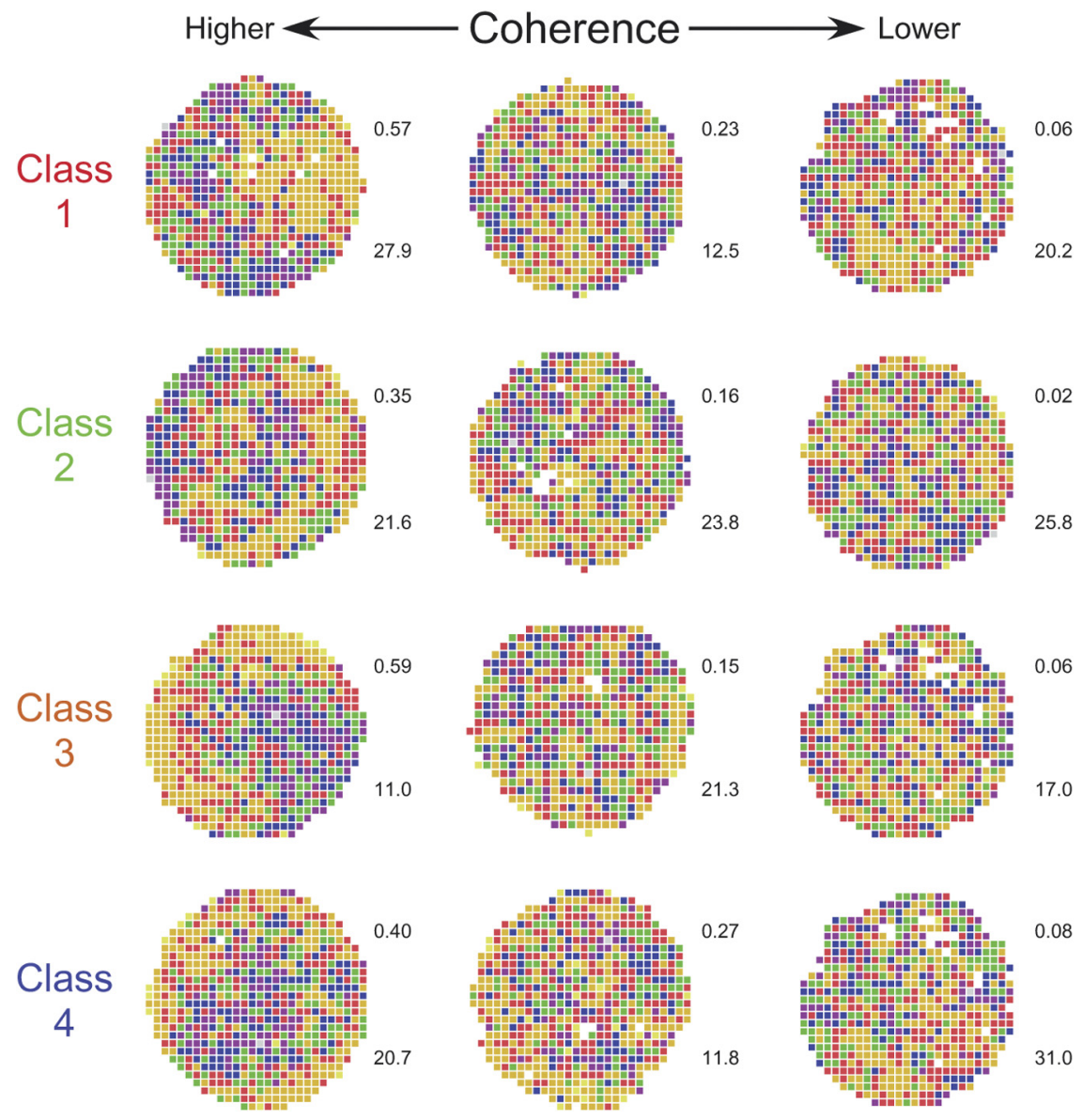

Figure 9. Color coded firing rate map examples for each of the four preferred phase classes. The example interneurons were chosen to illustrate the large spatial coherence range for each class, suggesting that there is not a strong differential contribution of the different classes to the great spatial firing specificity of place cells. (Spatial coherence was calculated as the z-transform of the firing rate correlation between each pixel and its neighbors — see Materials and Methods.) The number to the upper right of each map is the coherence (see Materials and Methods); the number to the bottom right of each map is the average firing rate for the entire recording session. to the difference between the periods of the two kinds of theta. Recently, however, differences have been noted in the relative spike timing of the same groups of interneurons recorded in the absence of drug and during urethane/ketamine/xylazine anesthesia (Senior et al., 2006). Accordingly, it would be very valuable to record from the same interneurons during anesthesia and free movement to allow direct comparisons of theta phase.

Lubenov and Siapas (2009) found that theta phase shifts along the mediolateral and rostrocaudal axes. In theory, this traveling wave property of hippocampal theta oscillation could introduce additional variance into our theta phase measurements. The magnitude of theta phase change was estimated to be $4-8^{\circ} / \mathrm{mm}$ in the CA1 region (Patel et al., 2009). Because fixed stereotaxic coordinates were used for electrode implantation, however, we estimate that at most $10^{\circ}$ of phase uncertainty might be produced the traveling of theta waves. In addition, Lubenov and Siapas (2009) showed that spike activity also exhibits a systematic phase advance that corresponds to the phase shift of theta oscillation. Therefore, this source of bias was eliminated by using theta recorded from the same tetrode as unit firing.

At the outset of our experiments, we imagined that interneurons with certain preferred theta phases would have stronger spatial firing rate variations than interneurons in other phase classes. In this view, the role of sculpting place cell firing fields would be played by specialized interneuron classes could not find clear distinctions among subtypes and therefore confine our analysis to classification according to theta phase.

Our starting point is a recent series of elegant studies that established a clear relationship between an interneuron's anatomical identity and its firing probability as a function of the phase of urethane-induced theta (Klausberger et al., 2003, 2004, 2005). Using juxtacellular staining after recording, five interneuron subtypes were recognized according to cell body location, arborization of neurites, and expression of specific chemical markers. When theta phase histograms were constructed for each subtype, they fell into four separable groups.

This phase separation predicts the four modes seen in the overall theta phase histogram for interneurons in alert rats. In addition, by assuming that the phase sequences in the two theta types are the same, we find a good correspondence between (1) class 1 and bistratified/O-LM cells and (2) class 3 and axo-axonic cells (Fig. 11). Admittedly, the matches between (1) class 2 and CCK cells and (2) class 4 and PV-immunopositive basket cells are weaker, but a variety of explanations are possible. For instance, if the PV and CCK basket cells are not purely phase locked but show partial time locking to the positive theta peak, they would fire at phases closer to this peak during the slower anesthetized theta.

In the simplest case, the differences in phase for each of the four cell classes in urethane and freely moving rats would reflect modifications of interneuronal activity relationships secondary with higher spatial firing precision. Such an outcome would have been conducive to building models of how location-specific firing is generated and modulated by sets of interacting interneurons and primary cells.

In fact, we saw no evidence from coherence or other signaling measures that the degree of spatial organization of interneurons is distributed unevenly across theta phase class. An attractive explanation for this lack is that cells from all of the theta phase classes form functional ensembles of two kinds. Ensembles of the first kind may be directly involved in allowing a fraction of pyramidal cells to show the location-specific firing characteristic of place cells. Such interneurons may play a crucial role in determining the precise membership of the active subset of pyramidal cells in a given environment (Muller et al., 1991). We imagine that interneurons in ensembles of this type have high coherence. By contrast, ensembles of the second type may function to prevent discharge of the silent pyramidal cells of the inactive subset, the complement of the active subset in a particular environment. This view motivates recording the same interneurons in two or more environments for which place cells show complete remappings. The strong prediction is that the coherence of individual interneurons would not be a cell-specific property but rather would vary from environment to environment. Moreover, by recording sufficient numbers of interneurons simultaneously in multiple environments, groupings might be found such that the 


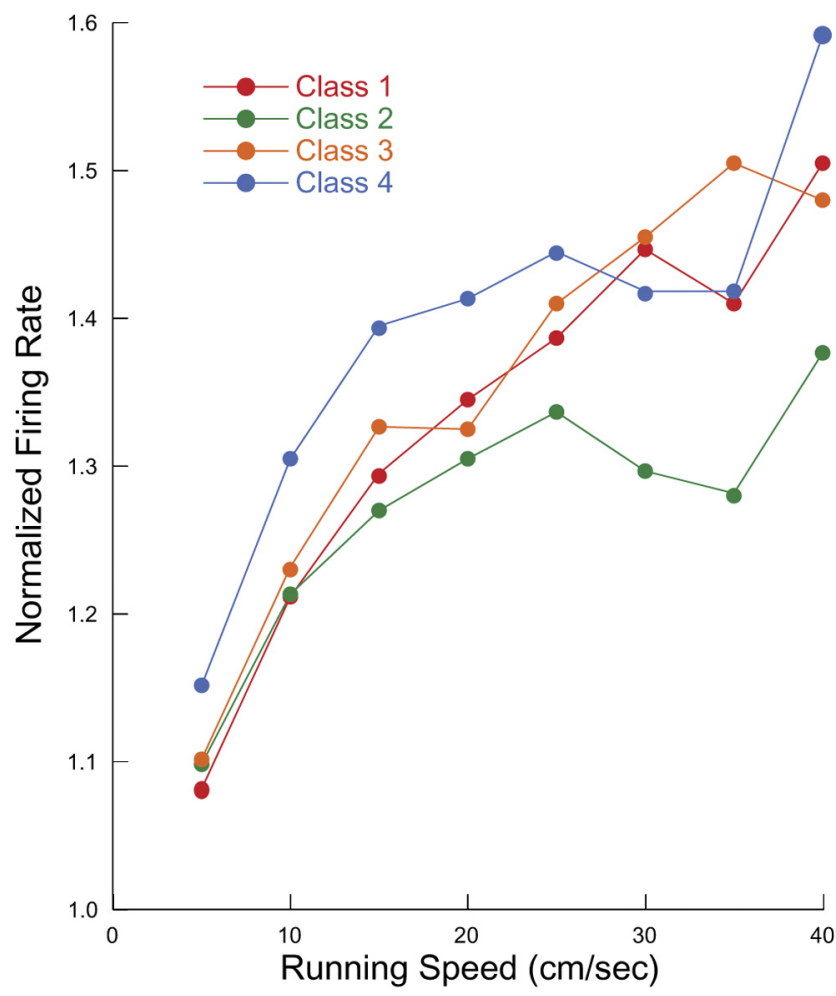

Figure 10. Normalized firing rate as a function of running speed for the four thetamodulated classes; the color code is the same as in Figure 7. Firing rate increases with running speed for each class, but the lack of a significant class by rate interaction from an ANOVA (see text) indicates that the functions are indistinguishable.

coherence of their members would covary. This would suggest that interneuron ensembles have a real identity and that they serve as critical components of cell assemblies (Harris et al., 2003).

A separate line of investigation arising from our results involves neural modeling in which a network set up to generate urethane theta would be tested for its ability to also generate the fast theta seen in freely moving rats. The network wiring would conform to current data (Klausberger and Somogyi, 2008), the fundamental frequency in urethane theta would be imposed from a pacemaker external to the network, and connection strengths would be adjusted so as to mimic the observed field potential rhythm and reproduce theta phase preferences. The pacemaker frequency would then be increased to $\sim 8 \mathrm{~Hz}$ to ask whether unmodified wiring would also generate walking theta and the associated phase preferences.

A final issue about theta phase preferences concerns possible differences in network organization during atropine-resistant versus atropine-sensitive theta (Kramis et al., 1975). Atropineresistant theta is seen during walking and other behaviors in which the entire rat moves or shifts its posture, whereas atropinesensitive theta is seen during quiet alertness and just before sudden motions (Kramis et al., 1975). It would be of great interest to ask whether theta phase preferences were invariant to the type of theta and whether such preferences changed during the very high $(>10 \mathrm{~Hz})$ frequency theta seen during sudden motions (Vanderwolf, 1969; Lenck-Santini et al., 2008).

\section{Theta-independent interneurons}

In addition to interneurons with clear theta phase preferences, we saw a second type whose autocorrelograms revealed no modulation at either theta or gamma frequency. These cells were quite

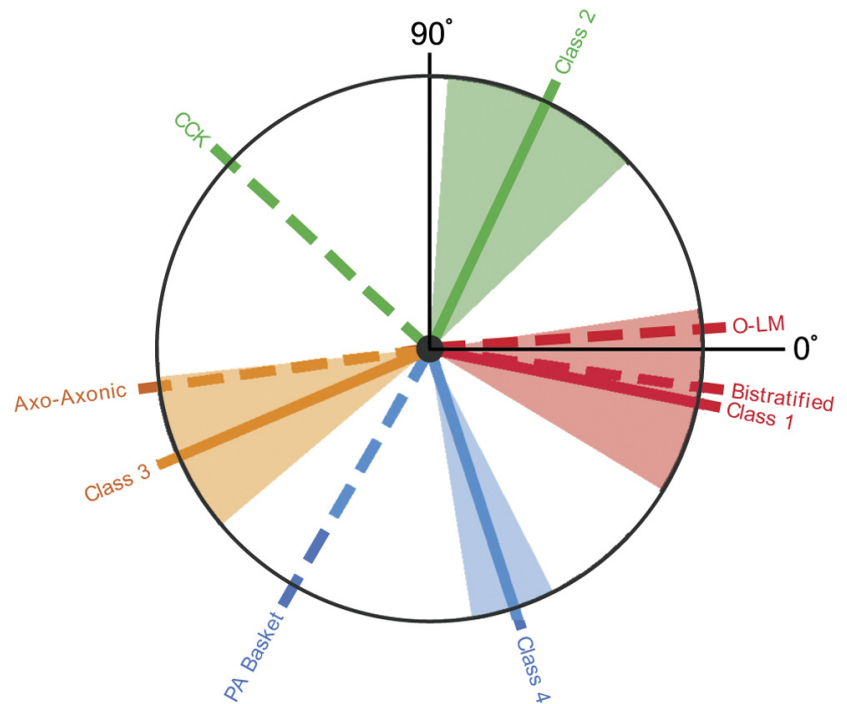

Figure 11. Proposed match between the preferred theta phase classes seen in freely moving rats and those seen in urethane-anesthetized rats; the color code for phase class is the same as in Figure 7. The phase coordinate is $0^{\circ}$ at 3:00 and increases counterclockwise. Theta phase classes in freely moving rats are indicated with solid lines; the urethane classes are indicated with dashed lines. The angle subtended by each of the colored sectors reflects the dispersion of the distribution for the corresponding phase preference class in freely moving rats. The urethane theta phase classes are as follows: bistratified, oriens/lacunosum-moleculare (0-LM), cholecystokinin-expressing (CCK), axo-axonic, and parvalbumin basket cells.

common, comprising one-third of our interneuron sample. Although hippocampal neurons whose activity is not theta modulated were noted earlier (García-Sánchez et al., 1978), these were generally believed to be principal cells (for review, see Vertes and Kocsis, 1997). Moreover, extensive studies of theta-modulated interneurons (Klausberger and Somogyi, 2008) did not focus on theta-independent interneurons, so that such cells seem to comprise a newly identified functional class.

In several regards, the properties of theta-independent cells were similar to theta-modulated interneurons, including increased rate during theta compared to LVF activity and more rapid firing during faster running. On the other hand, we found that these cells tended to occur in more superficial layers of CA1, with most recorded in the alveus and stratum oriens. On the assumption that extracellular spike signals are usually generated by cell bodies, possible candidates for the anatomical type of theta-independent cells are shown as classes 15-18 in Figure 1 of Klausberger and Somogyi (2008).

A prominent discharge feature of theta-independent cells is their tendency to fire high-frequency $(\sim 500 \mathrm{~Hz})$ trains of three or more spikes. In this way, they are reminiscent of the "bursty" cells described by Csicsvari et al. (1999), although their firing rates are higher and they occur with higher probability than bursty cells. Clues as to the functional role for this class of cells are likely to come from determining the extent and conditions under which high-frequency bursts are synchronous across individual cells. We did not record from enough pairs of theta-independent cells to ask whether the bursts are in register.

\section{Summary}

The most interesting aspects of our results are the existence in freely moving rats of four classes of theta-modulated interneurons whose preferred phases are quite close to those seen in anatomically identified interneurons during urethane anesthesia (Klausberger and Somogyi, 2008) and the lack of difference in the 
degree of spatial firing organization. The first finding suggests that theta during urethane anesthesia is indeed a valuable model for walking theta. The second finding limits the possible arrangements whereby interneurons cooperate in setting which pyramidal cells actively represent a given environment and the specific firing fields of the active place cells.

\section{References}

Akaike H (1974) A new look at the statistical model identification. IEEE Trans Automat Contr 19:716-723.

Amaral DG, Wittner L (1995) Hippocampal formation. In: The rat nervous system (Paxinos G, ed), pp 443-493. San Diego: Academic.

Baude A, Bleasdale C, Dalezios Y, Somogyi P, Klausberger T (2007) Immunoreactivity for the GABAA receptor alphal subunit, somatostatin and Connexin36 distinguishes axoaxonic, basket, and bistratified interneurons of the rat hippocampus. Cereb Cortex 17:2094-2107.

Buzsáki G, Grastyan E, Haubenreiser H, Czopf J, Kellenyi L (1982) Hippocampal slow wave activity: sources of controversy. In: Neuronal plasticity and memory formation (Marsan CA, Matties H, eds), pp 511-529. New York: Raven.

Csicsvari J, Hirase H, Czurkó A, Mamiya A, Buzsáki G (1999) Oscillatory coupling of hippocampal pyramidal cells and interneurons in the behaving rat. J Neurosci 19:274-287.

Czurkó A, Hirase H, Csicsvari J, Buzsáki G (1999) Sustained activation of hippocampal pyramidal cells by 'space clamping' in a running wheel. Eur J Neurosci 11:344-352.

Fenton AA, Csizmadia G, Muller RU (2000) Conjoint control of hippocampal place cell firing by two visual stimuli. I. The effects of moving the stimuli on firing field positions. J Gen Physiol 116:191-209.

Ferraguti F, Klausberger T, Cobden P, Baude A, Roberts JD, Szucs P, Kinoshita A, Shigemoto R, Somogyi P, Dalezios Y (2005) Metabotropic glutamate receptor 8-expressing nerve terminals target subsets of GABAergic neurons in the hippocampus. J Neurosci 25:10520-10536.

Fisher NI (1993) A test for the number of components in a mixture of von Mises distributions. In: Statistical analysis of circular data, Chap 4.6.2, pp 100-102. Cambridge, UK: Cambridge UP.

Fox SE, Ranck JB Jr (1981) Electrophysiological characteristics of hippocampal complex-spike cells and theta cells. Exp Brain Res 41:399-410.

Freund TF, Buzsáki G (1996) Interneurons of the hippocampus. Hippocampus 6:347-470.

Fuentealba P, Begum R, Capogna M, Jinno S, Márton LF, Csicsvari J, Thomson A, Somogyi P, Klausberger T (2008) Ivy cells: a population of nitric-oxideproducing, slow-spiking GABAergic neurons and their involvement in hippocampal network activity. Neuron 57:917-929.

García-Sánchez JL, Buño W Jr, Fuentes J, García-Austt E (1978) Nonrhythmical hippocampal units, theta rhythm and afferent stimulation. Brain Res Bull 3:213-219.

Hafting T, Fyhn M, Molden S, Moser M-B, Moser EI (2005) Microstructure of a spatial map in the entorhinal cortex. Nature 436:801-806.

Harris KD, Henze DA, Csicsvari J, Hirase H, Buzsáki G (2000) Accuracy of tetrode spike separation as determined by simultaneous intracellular and extracellular measurements. J Neurophysiol 84:401-414.

Harris KD, Csicsvari J, Hirase H, Dragoi G, Buzsáki G (2003) Organization of cell assemblies in the hippocampus. Nature 424:552-556.

Huxter JR, Zinyuk LE, Roloff EL, Clarke VR, Dolman NP, More JC, Jane DE, Collingridge GL, Muller RU (2007) Inhibition of kainate receptors reduces the frequency of hippocampal theta oscillations. J Neurosci 27:2212-2223.

Huxter JR, Allen K, Senior T, Dupret D, Pleydell-Bouverie B, Csicsvari J (2008) Rhythmic entrainment of hippocampal neurons at theta and gamma frequencies. FENS Abstr 4:128.15.

Jinno S, Klausberger T, Marton LF, Dalezios Y, Roberts JD, Fuentealba P, Bushong EA, Henze D, Buzsáki G, Somogyi P (2007) Neuronal diversity in GABAergic long-range projections from the hippocampus. J Neurosci 27:8790-8804.

Jung MW, McNaughton BL (1993) Spatial selectivity of unit activity in the hippocampal granular layer. Hippocampus 3:165-182.

Klausberger T, Somogyi P (2008) Neuronal diversity and temporal dynamics: the unity of hippocampal circuit operations. Science 321:53-57.

Klausberger T, Magill PJ, Márton LF, Roberts JD, Cobden PM, Buzsáki G, Somogyi P (2003) Brain-state- and cell-type-specific firing of hippocampal interneurons in vivo. Nature 421:844-848.
Klausberger T, Márton LF, Baude A, Roberts JD, Magill PJ, Somogyi P (2004) Spike timing of dendrite-targeting bistratified cells during hippocampal network oscillations in vivo. Nat Neurosci 7:41-47.

Klausberger T, Marton LF, O’Neill J, Huck JH, Dalezios Y, Fuentealba P, Suen WY, Papp E, Kaneko T, Watanabe M, Csicsvari J, Somogyi P (2005) Complementary roles of cholecystokinin- and parvalbumin-expressing GABAergic neurons in hippocampal network oscillations. J Neurosci 25:9782-9793.

Kramis R, Vanderwolf CH, Bland BH (1975) Two types of hippocampal rhythmical slow activity in both the rabbit and rat: relations to behavior and the effects of atropine, diethyl ether, urethane and pentobarbital. Exp Neurol 49:58-85.

Kubie JL, Muller RU, Bostock E (1990) Spatial firing properties of hippocampal theta cells. J Neurosci 10:1110-1123.

Lenck-Santini PP, Fenton AA, Muller RU (2008) Discharge properties of hippocampal neurons during performance of a jump avoidance task. J Neurosci 28:6773-6786.

Lubenov EV, Siapas AG (2009) Hippocampal theta oscillations are travelling waves. Nature 459:534-539.

McBain CJ, Fisahn A (2001) Interneurons unbound. Nat Rev Neurosci 2:11-23.

Mott DD, Dingledine R (2003) Interneuron diversity series: interneuron research—challenges and strategies. Trends Neurosci 26:484-488.

Muller RU, Kubie JL, Ranck JB Jr (1987) Spatial firing patterns of hippocampal complex-spike cells in a fixed environment. J Neurosci 7:1935-1950.

Muller RU, Kubie JL, Bostock EM, Taube JS, Quirk GJ (1991) Spatial firing correlates of neurons in the hippocampal formation of freely moving rats. In: Brain and space (Paillard J, ed), pp 296-333. Oxford: Oxford UP.

O'Keefe J (1979) A review of the hippocampal place cells. Prog Neurobiol 13:419-439.

O'Keefe J, Nadel L (1978) The hippocampus as a cognitive map. Oxford: Clarendon.

Olypher AV, Lánský P, Muller RU, Fenton AA (2003) Quantifying locationspecific information in the discharge of rat hippocampal place cells. J Neurosci Methods 127:123-135.

Patel J, Royer S, Buzsáki G (2009) Electrophysiological characteristics of the hippocampal circuits along the septo-temporal axis in the behaving rat. Soc Neurosci Abstr 35:192.10.

Paxinos G, Watson C (1998) The rat brain in stereotaxic coordinates. Sydney: Academic.

Rivard B, Li Y, Lenck-Santini PP, Poucet B, Muller RU (2004) Representation of objects in space by two classes of hippocampal pyramidal cells. J Gen Physiol 124:9-25.

Schwarz GE (1978) Estimating the dimension of a model. Ann Statist 6:461-464.

Senior TJ, O’Neill J, Klausberger T, Csicsvari J (2006) Hippocampal theta oscillations in the anaesthetised and drug-free rat. FENS Abstr 3:A197.15.

Sharp PE (1996) Multiple spatial/behavioral correlates for cells in the rat postsubiculum: multiple regression analysis and comparison to other hippocampal areas. Cereb Cortex 6:238-259.

Skaggs WE, McNaughton BL, Gothard K, Markus E (1993) An information theoretic approach to deciphering the hippocampal code. In: Advances in neural information processing systems, Vol 5 (Hanson S, Cowan J, Giles C, eds), pp 1030-1037. San Mateo, CA: Morgan Kaufmann.

Somogyi P, Klausberger T (2005) Defined types of cortical interneuron structure space and spike timing in the hippocampus. J Physiol 562:9-26.

Taube JS, Muller RU, Ranck JB Jr (1990) Head-direction cells recorded from the postsubiculum in freely moving rats. II. Effects of environmental manipulations. J Neurosci 10:436-447.

Theodoridis S, Koutroumbas K (2009) Pattern recognition, pp 44-49. Oxford: Academic

Theodoridis S, Koutroumbas K (2010) An introduction to pattern recognition: a MATLAB approach, pp 11-19. Oxford: Academic.

Tukker JJ, Fuentealba P, Hartwich K, Somogyi P, Klausberger T (2007) Cell type-specific tuning of hippocampal interneuron firing during gamma oscillations in vivo. J Neurosci 27:8184-8189.

Vanderwolf CH (1969) Hippocampal electrical activity and voluntary movement in the rat. Electroencephalogr Clin Neurophysiol 26:407-418.

Vertes RP, Kocsis B (1997) Brainstem-diencephalo-septohippocampal systems controlling the theta rhythm of the hippocampus. Neuroscience 81:893-926. 